\title{
Transition-Metal-Substituted Indium Thiospinels as Novel Intermediate-Band Materials: Prediction and Understanding of Their Electronic Properties
}

\author{
P. Palacios, ${ }^{1,2}$ I. Aguilera, ${ }^{1}$ K. Sánchez, ${ }^{1}$ J. C. Conesa, ${ }^{2}$ and P. Wahnón ${ }^{1}$ \\ ${ }^{1}$ Instituto de Energía Solar and Departamento Tecnologías Especiales ETSI Telecomunicación, UPM, Ciudad Universitaria, \\ Madrid 28040, Spain \\ ${ }^{2}$ Instituto de Catálisis y Petroleoquímica, CSIC, Cantoblanco, 28049 Madrid, Spain
}

(Received 12 February 2008; published 23 July 2008)

\begin{abstract}
Results of density-functional calculations for indium thiospinel semiconductors substituted at octahedral sites with isolated transition metals $(M=\mathrm{Ti}, \mathrm{V})$ show an isolated partially filled narrow band containing three $t_{2 g}$-type states per $M$ atom inside the usual semiconductor band gap. Thanks to this electronic structure feature, these materials will allow the absorption of photons with energy below the band gap, in addition to the normal light absorption of a semiconductor. To our knowledge, we demonstrate for the first time the formation of an isolated intermediate electronic band structure through $M$ substitution at octahedral sites in a semiconductor, leading to an enhancement of the absorption coefficient in both infrared and visible ranges of the solar spectrum. This electronic structure feature could be applied for developing a new third-generation photovoltaic cell.
\end{abstract}

One possible way of enhancing the efficiency of photovoltaic (PV) cells, proposed in recent years, is the use of intermediate-band materials [1]. A relatively narrow band [the intermediate band (IB)] is located in these materials between two other bands (filled and empty, respectively) akin to the valence and conduction bands (VB and CB, respectively) of a semiconductor of band-gap width $E_{g}$. This allows an electron from the VB to be promoted to the $\mathrm{IB}$, and from the latter to the $\mathrm{CB}$, upon absorption of photons with energy below $E_{g}$, achieving the same total result as with one photon of energy $E_{g}$. The use of this material could allow high PV efficiencies with an ideal limit of as much as $63.1 \%$ (if $E_{g} \approx 2.0 \mathrm{eV}$ and the IB is optimally placed between the VB and the $\mathrm{CB}$ ), while that achievable with one normal absorbing semiconductor (with $E_{g} \approx 1.1 \mathrm{eV}$ ) is $40.7 \%$ [1].

The IB should be partially filled, to allow comparable rates for both low energy photon absorption processes, and should not overlap with either the CB or the VB to avoid a fast transfer to the IB, via thermalization, of the carriers excited in the VB and CB. A well-formed band with significant dispersion and delocalization is also needed rather than discrete localized levels that would favor nonradiative recombination; the minimum dopant concentrations necessary to fulfill this condition when forming the IB via doping have been estimated [2].

Several doped semiconductors have been proposed to form the appropriate IB materials. O-doped ( $\mathrm{Zn}, \mathrm{Mn}) \mathrm{Te}$ and $\mathrm{N}$-doped $\mathrm{Ga}(\mathrm{As}, \mathrm{P})$ have been claimed, on the basis of spectroscopic data interpreted with a band anticrossing model, to form IB structures [3,4], although full firstprinciples quantum calculations were not made. Previous works by the present authors have shown, using densityfunctional theory (DFT) calculations, that IB materials can come about when a light transition metal $(M)$ such as Ti or
Cr partially substitutes the Ga in semiconductors of families already used as absorbents in PV cells, such as GaAs or $\mathrm{GaP}[5,6]$ or the chalcopyrite $\mathrm{CuGaS}_{2}$ [7]. However, in these calculations the IB formed by the inclusion of $\mathrm{Cr}$ nearly overlaps the VB (and actually mixes with it, in the case of the chalcopyrite, if a DFT $+U$ approximation is used [7]); with $\mathrm{Ti}$ as the substituent, the calculations show an overlap between the IB and the CB which might disappear (or not) if advanced theoretical methods able to reproduce band gaps better than standard DFT are used.

The aim of this work is to show, for the first time, a system presenting an electronic structure that contains an isolated, partially filled narrow IB formed by substituting an atom in an octahedral environment with a $M$ atom. This IB contains three $t_{2 g}$-type states per $M$ atom instead of two $e_{g}$-type states formed in the tetrahedral environment of the aforementioned cases [5-7]. The former should be more stable thermodynamically as these $M$ 's normally prefer to be surrounded by six atoms rather than by four.

The proposed semiconductor family, which is not currently being used as the light absorber in PV devices, is indium-based thiospinels, $\operatorname{MgIn}_{2} \mathrm{~S}_{4}$ and particularly the defect spinel $\operatorname{In}_{2} \mathrm{~S}_{3}$, which has a band gap of an appropriate size (direct band gap of around $2.1 \mathrm{eV}[8,9]$ ) and a majority of its In atoms octahedrally coordinated. This octahedral geometry is expected to facilitate the desired substitution, as it is the coordination preferred by the $M$ ions of interest. $\mathrm{In}_{2} \mathrm{~S}_{3}$ has an added advantage as it is frequently used as a window layer in chalcopyrite-based PV cells, so that methods to prepare it in a convenient thin film form are known. We propose $\mathrm{Ti}$ and $\mathrm{V}$ as the best $M$ candidates for this purpose, as they may lead to a partially occupied IB by contributing (being formally in the trivalent state) with one or two electrons, respectively, per $M$ atom to the lowest spin-polarized manifold, containing three $t_{2 g}$-type states 
per $M$ atom, which will be split from the $3 d$ shell of the latter by the octahedral environment.

Our calculations were made using spin-polarized DFT with the generalized gradient (GGA) approximation and the Perdew-Wang 1991 functional [10]. The plane-wave VASP code [11] with projector augmented wave (PAW) potentials [12,13] was used. Unconstrained relaxations of ions and cells with a convergence tolerance of $0.01 \mathrm{eV} / \AA$ for atomic forces were made for all systems. From the resulting converged wave functions, total and projected density of states (PDOS), band diagrams, and optical properties were computed. To obtain absorption coefficients that can be compared with the experiment, a shift in the CB was applied; its magnitude, chosen to reproduce the experimental band gap of the undoped semiconductor, was retained for the $M$-doped systems. Then we calculated the real and imaginary parts of the dielectric function, as well as the absorption coefficient $[14,15]$. We considered only direct transitions.

Calculations have been made for primitive cells of crystalline structures based on a cubic spinel $\mathrm{MgIn}_{2} \mathrm{~S}_{4}$ (space group $F d-3 m$ ). Although this spinel is known to have some degree of inversion [16], we have made all of the calculations for a direct structure in the model for simplicity. One of the four octahedrally coordinated In atoms in the primitive cell is substituted by V or Ti. Similar calculations are carried out as well for structures based on the structure of $\operatorname{In}_{2} \mathrm{~S}_{3}$ [17], which crystallizes in the I41/amd space group. It can be considered as an ordered defect (super)structure of the aforementioned spinel. In this case, two of the twelve octahedrally coordinated In atoms in the primitive cell are substituted by $\mathrm{V}$ or $\mathrm{Ti}$ in well-separated, symmetry-equivalent positions. Calculations for the nonsubstituted host materials were also made.

For the pure host semiconductors, i.e., with no $M$ inserted, the calculations produce, after full geometry relaxation, unit cells with dimensions slightly above the experimental ones; the average In-S distances around the octahedral positions are also a bit higher than the experimental values (see Tables I and II). These deviations are typical for the GGA approach [7,19]. For the $\mathrm{MgIn}_{2} \mathrm{~S}_{4}$ spinel, the computed band-gap width is ca. $1.65 \mathrm{eV}$ and is of the direct type. As expected for a GGA calculation, this is lower than the experimental value of $2.1 \mathrm{eV}$ [20] but not as bad as it could be. This might be due to the use of a direct structure. For $\operatorname{In}_{2} \mathrm{~S}_{3}$, the calculated width of the band gap (also direct) is about $0.86 \mathrm{eV}$, while the experimental value is 2.1 [8].

After the substitution of In with the $M$ as mentioned above, the unit cell volumes decrease slightly; this can be connected to the (average) $M-S$ distances, found to be shorter than the In- $S$ distances in the parent compound as can be expected since the Ti and V atoms are smaller than the In atom. These $M-S$ distances are, however, higher than those in the pure sulphides of the corresponding $M$ in the
TABLE I. Lattice parameter $a$ (in $\AA$ ) and atomic distances $d(M-S)$ (in $\AA$ ) for the $\operatorname{In}_{2} \mathrm{~S}_{3}$ defect spinel and its substituted systems. In brackets appears the experimental result [17] and in braces the number of $M-S$ or In-S bonds presented in the structure.

\begin{tabular}{cccc}
\hline \hline & \multicolumn{1}{c}{$\mathrm{In}_{2} \mathrm{~S}_{3}$} & $\mathrm{In}_{14} \mathrm{Ti}_{2} \mathrm{~S}_{24}$ & $\mathrm{In}_{14} \mathrm{~V}_{2} \mathrm{~S}_{24}$ \\
\hline$a$ & $7.77(7.62)$ & 7.67 & 7.65 \\
$c / a$ & $4.24(4.26)$ & 4.31 & 4.35 \\
$d\left(\operatorname{In}_{\mathrm{oct}}-S\right)$ & $2.58(2.61)\{4\}$ & $2.58\{2\}$ & $2.60\{2\}$ \\
& $2.73(2.77)\{2\}$ & $2.69\{4\}$ & $2.70\{4\}$ \\
& $2.70(2.56-2.80)\{6\}$ & & \\
$d\left(\operatorname{In}_{\text {tet }}-S\right)$ & $2.53(2.43-2.48)$ & 2.52 & 2.52 \\
$d\left(M_{\mathrm{oct}}-S\right)$ & & $2.46\{2\}$ & $2.39\{2\}$ \\
& & $2.54\{4\}$ & $2.51\{4\}$ \\
\hline \hline
\end{tabular}

trivalent state, computed to be $2.455 \AA$ for $\mathrm{Ti}_{2} \mathrm{~S}_{3}$ (in an ordered version of the experimental rhombohedral lattice [21]) and 2.413 or $2.359 \AA$ for the structures of $\mathrm{V}_{3} \mathrm{~S}_{4}$ [22] or $\mathrm{V}_{5} \mathrm{~S}_{8}$ [23] (a stable well defined compound of stoichiometry $\mathrm{V}_{2} \mathrm{~S}_{3}$ has not been reported). Thus a coordination distance misfit exists in the substituted materials.

Figure 1 displays the total DOS and PDOS (projecting on the metal $3 d$ orbitals) computed for both $M$-substituted $\mathrm{In}_{14} \mathrm{M}_{2} \mathrm{~S}_{24}$ systems and the $\mathrm{In}_{2} \mathrm{~S}_{3}$ semiconductor. After full geometry relaxation in substituted systems, the electronic structure contains strongly spin-polarized levels that come about from the $3 d$ metal states. Both majority and minority spin levels appear to be split into two branches, as expected for $M$ atoms in an octahedral environment: a lower branch arising from the triply degenerated $t_{2 g}$ manifold and an upper one arising from the doubly degenerated $e_{g}$ manifold. For the majority spin states, the lower branch is crossed by the Fermi energy so that it contains one and two electrons per $M$ atom in the Ti and V cases, respectively (as expected from the $3 d^{1}$ and $3 d^{2}$ configurations of their trivalent states), while the upper branch appears inside the CB.

Figure 1 shows that the computed VB-CB band gaps of the $\operatorname{In}_{14} \mathrm{M}_{2} \mathrm{~S}_{24}$ systems are around $0.9 \mathrm{eV}$, close to that calculated for the undoped $\operatorname{In}_{2} \mathrm{~S}_{3}$, and that the lower branches of the new bands are located within this gap in different positions for the two M's. It is well known that

TABLE II. Lattice parameter $a$ (in $\AA$ ) and atomic distances $d(M-S)$ (in $\AA$ ) for the $\operatorname{MgIn}_{2} \mathrm{~S}_{4}$ spinel and its substituted systems. In brackets appears the experimental result [18] and in braces the number of $M-S$, In-S, or $\mathrm{Mg}-\mathrm{S}$ bonds presented in the structure.

\begin{tabular}{lccc}
\hline \hline & $\mathrm{MgIn}_{2} \mathrm{~S}_{4}$ & $\mathrm{Mg}_{2} \mathrm{In}_{3} \mathrm{TiS}_{8}$ & $\mathrm{Mg}_{2} \mathrm{In}_{3} \mathrm{VS}_{8}$ \\
\hline$a$ & $10.78(10.71)$ & 10.68 & 10.68 \\
$d(\mathrm{In}-S)$ & $2.63(2.58)$ & $2.61\{2\}-2.65\{4\}$ & $2.61\{2\}-2.64\{4\}$ \\
$d(\mathrm{Mg}-S)$ & $2.46(2.48)$ & $2.45\{1\}-2.47\{3\}$ & $2.44\{1\}-2.48\{3\}$ \\
$d(M-S)$ & & 2.51 & 2.48 \\
\hline \hline
\end{tabular}


(a)

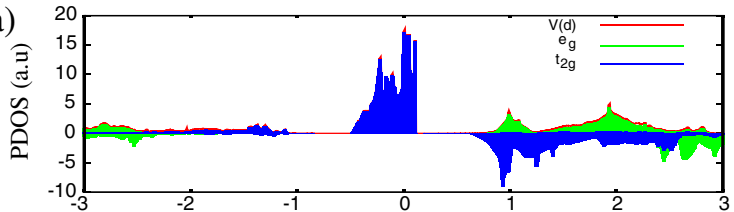

(b)
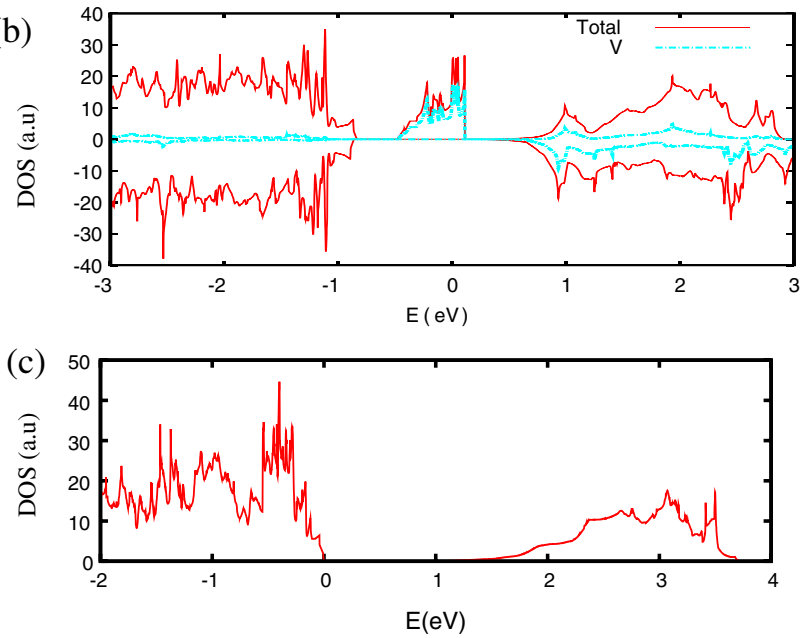

(d)

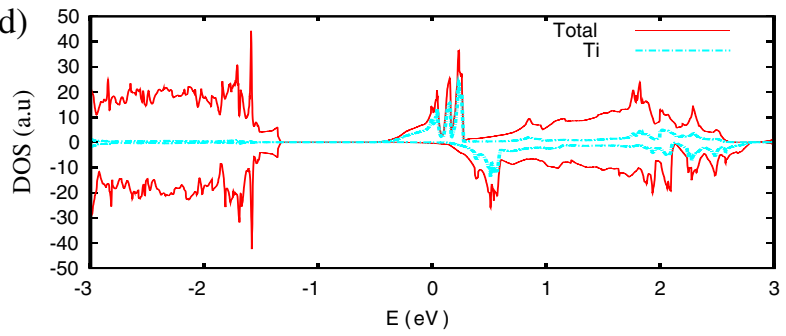

(e)

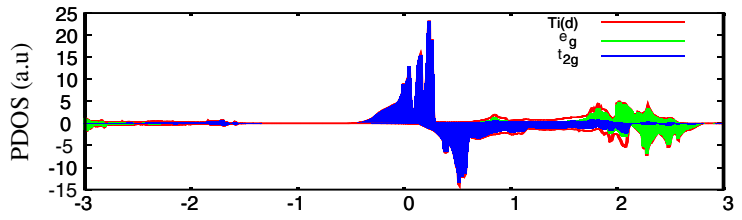

FIG. 1 (color online). DOS and PDOS obtained with GGA calculations for $\operatorname{In}_{14} \mathrm{M}_{2} \mathrm{~S}_{24}$ alloy materials and the $\mathrm{In}_{2} \mathrm{~S}_{3}$ host semiconductor. (a),(b) $M=V$, (c) $\operatorname{In}_{2} \mathrm{~S}_{3}$, and (d),(e) $M=\mathrm{Ti}$. Plots (b) and (d) show the total DOS and the partial $M$ contribution to the DOS; plots (a) and (e) show the splitting of the $M d$-orbitals manifold into $t_{2 g}$-and $e_{g}$-type components. The Fermi level is set to $E=0$.

GGA calculations underestimate band gaps, but this mainly affects the separation between the occupied and unoccupied levels; consequently, the energy difference between the VB and the IB is not expected to be affected by this underestimation $[6,24]$. However, the IB-CB real gap will be larger than that calculated here. To estimate its real value, a shift in the $\mathrm{CB}$ could be applied by an amount appropriate to reproduce the VB-CB gap in the original semiconductor. If this were done, one would obtain a band structure in which a real IB would appear with the desired characteristics. This will also occur even for the Ticontaining system, which in the GGA calculations appears to have the partially filled $t_{2 g}$-type band overlapping the CB slightly.

Figure 2 shows the DOS curve for the undoped $\mathrm{MgIn}_{2} \mathrm{~S}_{4}$ semiconductor and the band structure diagrams and the DOS and PDOS curves for the $\mathrm{Mg}_{2} \mathrm{In}_{3} \mathrm{MS}_{8}$ alloy systems; the decomposition of the latter in $t_{2 g}$ and $e_{g}$ contributions, which have similar features as in those in Fig. 1, are omitted here for simplicity. These band diagrams, simpler than those for the $\operatorname{In}_{14} \mathrm{M}_{2} \mathrm{~S}_{24}$ (omitted above for this reason), complement the understanding of the formation of the IB by showing its shape and how the direct character of the $\mathrm{MgIn}_{2} \mathrm{~S}_{4}$ VB-CB gap is maintained in the substituted systems. Furthermore, Figs. 2(a) and 2(b) clearly show similar shapes for the VB and the CB in both the doped and the undoped systems except for the states concerning the IB.

In $\mathrm{Mg}_{2} \mathrm{In}_{3} \mathrm{VS}_{8}$, the $t_{2 g}$-type spin-up band appears partially filled and separated from the VB and the $\mathrm{CB}$, as desired for the IB scheme; the $e_{g}$-type spin-up band with two levels is easily recognized within the $\mathrm{CB}$. In the spindown channel, as can be seen in the PDOS curve [Fig. 2(b), left panel], all of the new $d$ bands are empty and merged with the conduction band. The Fermi level lies $0.71 \mathrm{eV}$ above the VB and $1.035 \mathrm{eV}$ below the $\mathrm{CB}$, the VB-CB separation being close to that computed for the undoped semiconductor. The results for $\mathrm{Mg}_{2} \mathrm{In}_{3} \mathrm{TiS}_{8}$, displayed in Figs. 2(b) and 2(c) (right panels), show an electron filling pattern as in $\operatorname{In}_{14} \mathrm{Ti}_{2} \mathrm{~S}_{24}$, with one electron per $M$ atom in

(a)
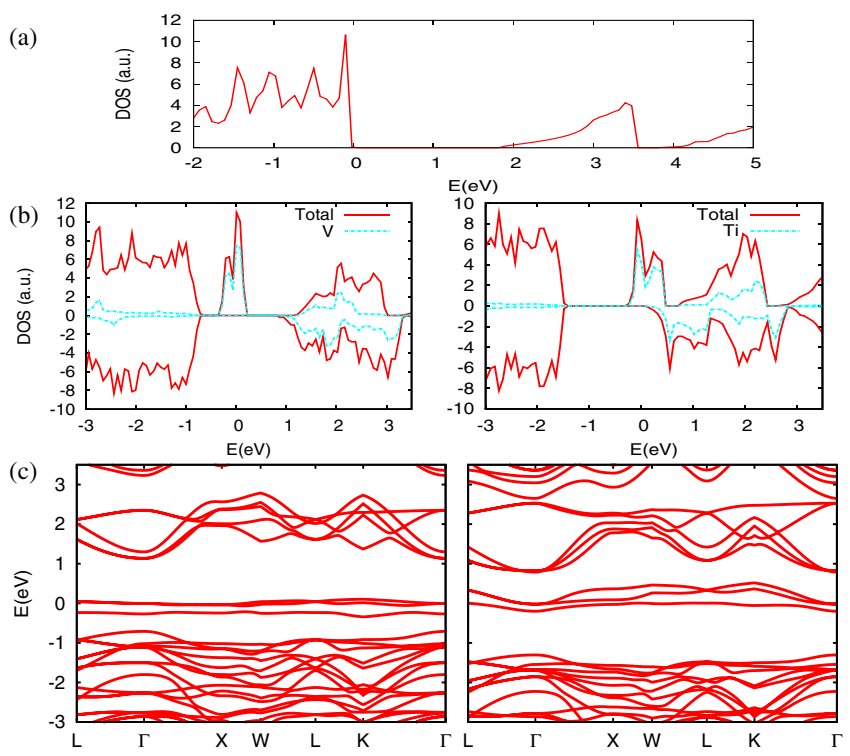

FIG. 2 (color online). DOS and PDOS curves obtained with GGA calculations for $\mathrm{Mg}_{2} \mathrm{In}_{3} \mathrm{MS}_{8}$ alloy materials and $\mathrm{MgIn}_{2} \mathrm{~S}_{4}$ host semiconductor. (a) $\mathrm{MgIn}_{2} \mathrm{~S}_{4}$, (b) $M=V$ (left panel) and $M=$ Ti (right panel). Plot (c) displays the spin-up band diagrams (including high symmetry $k$ points of the Brillouin zones labeled as corresponds to the semiconductor space groups) for the alloy system: $M=V$ (left panel) and $M=\mathrm{Ti}$ (right panel). The Fermi level is set to $E=0$. 


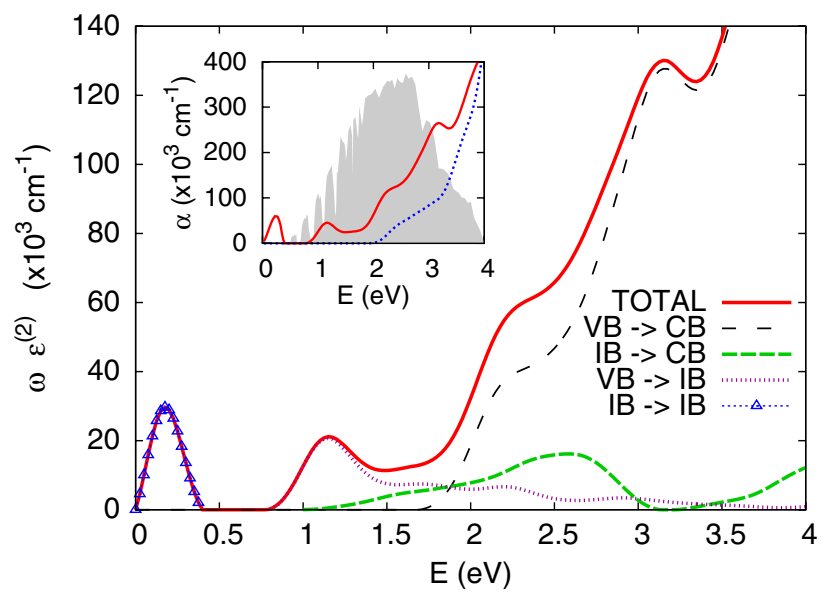

FIG. 3 (color online). $\omega$-weighted imaginary part $\epsilon^{(2)}$ of the total dielectric function (solid line) and the partial contribution of the different direct transitions to it, computed for the $\mathrm{Mg}_{2} \mathrm{In}_{3} \mathrm{VS}_{8}$ systems. The inset shows the optical absorption computed from the dielectric function for $\mathrm{Mg}_{2} \mathrm{In}_{3} \mathrm{VS}_{8}$ (solid line) and the semiconductor spinel $\mathrm{MgIn}_{2} \mathrm{~S}_{4}$ (dots).

the $t_{2 g}$ levels, these being closer to the CB than for vanadium but still isolated from it, thus forming a separate IB. Otherwise, the $d$-band shapes are qualitatively the same as in vanadium substitution. Note that the IB-CB separation is an indirect gap, from points $K$ to $\Gamma$, in both $\mathrm{Mg}_{2} \mathrm{In}_{3} \mathrm{MS}_{8}$ systems.

For the $\mathrm{Mg}_{2} \mathrm{In}_{3} \mathrm{VS}_{8}$ system, the optical properties were computed [25], and the results are displayed in Fig. 3. The optical absorption is compared to that calculated for the undoped semiconductor and seen to begin at almost zero energy, being significant in almost all of the energy range up to the UV region. In order to determine which transitions are responsible for the absorption peaks, the partial contribution of each transition to the imaginary part of the dielectric function is also presented. The first peak at the lowest energy is due to transitions within the IB. The contribution of the transitions from the VB to the IB starts at $E \approx 0.7 \mathrm{eV}$ and that from the IB to the $\mathrm{CB}$ at $E \approx$ $1.1 \mathrm{eV}$. Finally, the main contribution, i.e., the usual transition from the $\mathrm{VB}$ to the $\mathrm{CB}$, is seen starting at $E \approx$ $1.8 \mathrm{eV}$. This confirms the enhancement in the optical absorption resulting from the metal-derived IB.

In summary, we present with first-principles calculations, the structural, electronic, and optical properties of new $M$-substituted In thiospinel compounds presenting a narrow half-filled intermediate band isolated from the VB and the CB of the host semiconductor. The influence of the octahedral environment around the metal becomes crucial in obtaining the right features of this band. The computed optical absorption of these compounds compared to the corresponding undoped semiconductor predicts a significant absorption below the band gap of the parent semiconductor and an enhancement of the optical absorption in the whole energy range of the visible region. Moreover, these $\mathrm{In}_{2} \mathrm{~S}_{3}$ derivatives, for which the technology to grow them as thin films is already known, seem to be promising systems for developing more efficient novel optoelectronic devices. Their experimental synthesis is at present underway.

This work was funded by the FULLSPECTRUM project (SES-CT-2003-502620), by the 6th European Programme, and by the GENESIS-FV(CSD2006-0004) and the CALIBAND(MAT2006-10618) projects of the Spanish MEC and from the Community of Madrid NUMANCIAMA(S-05050/ENE/0310) project. The computer resources provided by the Madrid Supercomputing Center (CeSViMa) are acknowledged.

[1] A. Luque and A. Martí, Phys. Rev. Lett. 78, 5014 (1997).

[2] A. Luque, A. Martí, E. Antolín, and C. Tablero, Physica (Amsterdam) 382B, 320 (2006).

[3] K. M. Yu et al., J. Appl. Phys. 95, 6232 (2004).

[4] K. M. Yu et al., Appl. Phys. Lett. 88, 092110 (2006).

[5] P. Wahnón and C. Tablero, Phys. Rev. B 65, 165115 (2002); C. Tablero and P. Wahnón, Appl. Phys. Lett. 82, 151 (2003).

[6] P. Wahnón et al., J. Mater. Sci. 40, 1383 (2005); P. Palacios et al., Phys. Rev. B 73, 085206 (2006).

[7] P. Palacios et al., Thin Solid Films 515, 6280 (2007).

[8] N. Barreau et al., Thin Solid Films 431-432, 326 (2003).

[9] J. Herrero and J. Ortega, Sol. Energy Mater. 17, 357 (1988).

[10] J. Perdew et al., Phys. Rev. B 46, 6671 (1992).

[11] G. Kresse and J. Hafner, Phys. Rev. B 47, R558 (1993).

[12] G. Kresse and D. Joubert, Phys. Rev. B 59, 1758 (1999). All PAW potentials used, from the VASP library, are obtained by the method explained in this reference.

[13] P. E. Blöchl, Phys. Rev. B 50, 17953 (1994).

[14] B. Adolph, J. Furthmüller, and F. Bechstedt, Phys. Rev. B 63, 125108 (2001).

[15] M. Gajdoš et al., Phys. Rev. B 73, 045112 (2006).

[16] M. Wakaki et al., Jpn. J. Appl. Phys. 21, 958 (1982).

[17] N. Rampersadh, A. Venter, and D. Billing, Physica (Amsterdam) 350B, E383 (2004).

[18] H. Hahn and W. Klingler, Z. Anorg. Allg. Chem. 263, 177 (1950).

[19] J. J. Fernández, C. Tablero, and P. Wahnón, Comput. Mater. Sci. 28, 274 (2003).

[20] P. M. Sirimanne, N. Sonoyama, and T. Sakata, J. Solid State Chem. 154, 476 (2000).

[21] M. Onoda and M. Saeki, Chem. Lett. 9, 665 (1980).

[22] C. Mujica, J. Llanos, and O. Wittke, J. Alloys Compd. 226, 136 (1995).

[23] W. Bensch and J. Koy, Inorg. Chim. Acta 206, 221 (1993).

[24] P. Wahnón et al., in Proceedings of the 20th European Photovoltaic Solar Energy Conference (WIP-Renewable Energies, Munich, 2007), ISBN-3-936338-22-1, p. 56.

[25] I. Aguilera, P. Palacios, and P. Wahnón, Thin Solid Films 516, 7055 (2008). 